Arq. Bras. Med. Vet. Zootec., v.72, n.6, p.2101-2110, 2020

\title{
Anatomia do sistema reprodutor feminino de Alouatta belzebul (Linnaeus, 1766)
}

[Anatomy of the female reproductive system of the Alouatta belzebul (Linnaeus, 1766)]

\author{
E.R. Pereira1, V.C.M.C. Pires ${ }^{1}$, R.J. Fernandes ${ }^{1}$, D.K.S. Pereira ${ }^{2}$, F.R. Melo ${ }^{3}$, \\ R.K.W. Schell ${ }^{4}$, A.C. Zarpelon-Schutz ${ }^{4}$, K.F. Pereira ${ }^{4}$
}

${ }^{1}$ Faculdade Morgana Potrich - Mineiros, GO

${ }^{2}$ Centro Universitário da Fundação Assis Gurgacz - Cascavel, PR

${ }^{3}$ Universidade Federal de Viçosa - Viçosa, MG

${ }^{4}$ Universidade Federal do Paraná - Campus Toledo, PR

\begin{abstract}
RESUMO
O conhecimento da anatomia de qualquer animal silvestre é de fundamental importância para sua preservação e proteção. Neste contexto, o presente estudo objetivou descrever a morfologia do sistema reprodutor feminino deAlouatta belzebul. Foram utilizados seis espécimes de A. belzebul, fêmeas, adultas, e livres de lesões. Observou-se macroscopicamente que os ovários têm características morfológicas em formato ovoides, com superfície lisa, e, na análise histológica na região de córtex, evidenciou-se folículos ovarianos em diferentes estágios de desenvolvimento. As tubas uterinas anatomicamente são finas e curvilíneas, apresentando uma camada mucosa, uma muscular e outra serosa. O útero possui formato simples, com fundo globoso, com um miométrio altamente vascularizado, sendo organizado em feixes de fibras musculares lisas. A estrutura anatômica da vagina apresentou-se como um tubo muscular longo de paredes finas, onde, na região vestibular, o óstio externo da uretra é marcado por uma papila uretral bilobada e, na região de vulva, em sua porção caudal, contatou-se um clitóris bem desenvolvido. No que concerne à análise histológica da vagina, verificou-se, em região de mucosa vaginal, um extrato basal composto por epitélio estratificado pavimentoso não queratinizado atrófico. As descrições morfológicas fornecem, de forma inédita, informações importantes relativas à anatomia macroscópica e microscópica do sistema reprodutor feminino dessa espécie.
\end{abstract}

Palavras-chave: bugio, morfologia, órgãos genitais femininos, primatas

\begin{abstract}
Knowledge of the anatomy of any wild animal is of fundamental importance for its preservation and protection. In this context the present study aimed to describe the morphology of the female reproductive system of A. belzebul. We used 6 specimens of A. belzebul, female, adult and free of lesions. It was macroscopically observed that the ovaries are ovoid with smooth surface and the histological analysis in cortical region showed ovarian follicles in different stages of development. The fallopian tubes are anatomically thin and curvilinear, with one mucous layer, one muscular and one serous layer. The uterus was presented in a simple format with a globular fundus, with a highly vascularized myometrium, being organized in bundles of smooth muscle fibers. The anatomical structure of the vagina presented itself as a long thin-walled muscular tube where in the vestibular region the external orifice of the urethra is marked by a bilobed urethral papilla and in the caudal portion in its caudal portion a well-developed clitoris. Regarding the histological analysis of the vagina, a basal extract composed of atrophic non-keratinized stratified squamous epithelium was found in the vaginal mucosa region. The morphological descriptions provide important information regarding the macroscopic and microscopic anatomy of the female reproductive system of this species in an unprecedented way.
\end{abstract}

Keywords: bugio, morphology, female genital organisms, primates

Recebido em 29 de junho de 2019

Aceito em 9 de junho de 2020

E-mail: ericarezende@fampfaculdade.com.br 


\section{INTRODUÇÃO}

Os primatas neotropicais apresentam variações em seus aspectos reprodutivos, e suas particularidades demonstram a grande adaptabilidade aos mais diversos ambientes e situações, respondendo a esses desafios da evolução com múltiplos sistemas de acasalamento, diferentes tipos de ciclos ovarianos e mecanismos de ovulação (Guimarães, 2007).

Os primatas do Velho Mundo apresentam, em sua maioria, ciclos ovarianos do tipo menstrual, semelhante ao descrito em humanos do gênero feminino, com ovulações espontâneas e comportamento de cópula ocorrendo em qualquer fase do ciclo e em qualquer época do ano. Em relação aos primatas do Novo Mundo, é possível identificar tanto ciclos ovarianos do tipo menstrual quanto ciclos estrais. Em Sapajus libidinosus, o ciclo ovariano é do tipo menstrual e, em Callithrix jacchus, o ciclo ocorre de forma estral, permitindo que a espécie possa copular em qualquer fase do ciclo e, inclusive, possa realizar cópulas férteis no período pós-parto e durante a lactação (Dixon, 1998).

O sistema reprodutor feminino humano (ou sistema genital feminino) é classificado em órgãos internos e externos. Os órgãos genitais femininos internos são denominados: vagina, útero, ovários e tubas uterinas. E o termo vulva, ou pudendo, refere-se aos órgãos genitais femininos externos, que são denominados de: monte púbico, lábios maiores, lábios menores, clitóris e bulbo do vestíbulo (Dangelo; Fattini, 2011). Pesquisas atuais na área biomédica têm sido desenvolvidas tanto em primatas neotropicais como em primatas do Velho Mundo, com uma grande importância devido à similaridade desses com o humano. Vários avanços em medicina humana já foram alcançados com o estudo de primatas (Dixson, 1994; Bennett et al., 1995), sendo a reprodução de primatas um dos ramos de pesquisas que mais contribui com o conhecimento sobre as espécies e a sua preservação.

Esse sistema reprodutor pode vir a apresentar variações anatômicas em relação à morfologia dos órgãos genitais femininos entre diferentes espécies de primatas. Poucos dados são encontrados na literatura sobre a anatomia do sistema reprodutor feminino de primatas, sendo necessários estudos detalhados dos órgãos reprodutores, o que justifica este trabalho com a espécie de Alouatta belzebul.

\section{MATERIAL E MÉTODOS}

Para esta pesquisa, foram disponibilizados seis espécimes de A. belzebul, fêmeas, adultas, com idades aproximadas entre quatro e cinco anos e livres de lesões. Esses animais foram cedidos pelo Projeto de Salvamento e Aproveitamento Científico da Fauna (PSACF) - UHE Belo Monte, no estado do Pará (PA). Os animais vieram a óbito no Centro de Triagem de Animais Silvestres do empreendimento, foram congelados e posteriormente doados para a Universidade Federal de Jataí (UFJ). O projeto foi aprovado pelo Comitê de Ética em Experimentação Animal (Ceua), com protocolo $\mathrm{n}^{\circ} 37 / 2018$.

Para a anatomia macroscópica, os animais foram previamente fixados, com perfusão de solução aquosa de formol a $10 \%$ através da artéria carótida comum direita, abordada por incisão cervical mediana ventral. Em seguida, os espécimes foram submersos em recipientes de solução aquosa de formol a $10 \%$ e mantidos em tanque fechado e opaco para a dissecação. Após um período de 72 horas, os espécimes foram retirados do tanque e dissecados. Realizou-se uma incisão na linha mediana ventral para abertura da cavidade abdominal rebatendo-se a pele e outras camadas de tecidos, a fim de facilitar o acesso aos órgãos genitais.

Após a secção da cavidade pélvica, foi realizada uma incisão na região da sínfise púbica e a total remoção dos órgãos genitais, para que seus componentes pudessem ser analisados e descritos individualmente. A mensuração dos órgãos do sistema reprodutor feminino foi feita com auxílio de um paquímetro (Mitutoyo ${ }^{\circledR}$ ). As medidas objetivaram a verificação de largura, comprimento e altura e foram documentadas em câmera fotográfica do celular Iphone XR (12 megapíxeis). A denominação das estruturas foi baseada na Nomina Anatomica Veterinaria internacional de 2017.

Para a anatomia microscópica, foram utilizadas amostras dos órgãos genitais femininos de três espécimes de Alouatta belzebul. As amostras foram processadas com o uso de técnicas histológicas de rotina e coradas com hematoxilina e eosina (HE), sendo os cortes analisados em 
microscópios ópticos. As fotomicrografias para documentação foram obtidas em microscópio óptico Nikon E-100.

\section{RESULTADOS E DISCUSSÃO}

O sistema reprodutor feminino da espécie $A$. belzebul é composto por órgãos internos constituídos por um par de ovários, um par de tubas uterinas, útero, vagina e a vulva como órgão externo, conforme evidenciado na Fig.1.
Em A. belzebul, os ovários apresentam formato ovoide e estão fixados pelos ligamentos mesovário e mesossalpinge às tubas uterinas, bilateralmente ao útero e situados na fossa ovárica. Cada ovário apresenta uma face medial e outra lateral, uma extremidade tubária e outra uterina e uma borda livre e outra mesovariana. As medidas do comprimento dos ovários variaram de 0,6 a $1,4 \mathrm{~cm}$. A largura variou de 0,5 a $1,1 \mathrm{~cm}$, e sua altura de 0,5 a $1,0 \mathrm{~cm}$, conforme indica a Tab. 1.

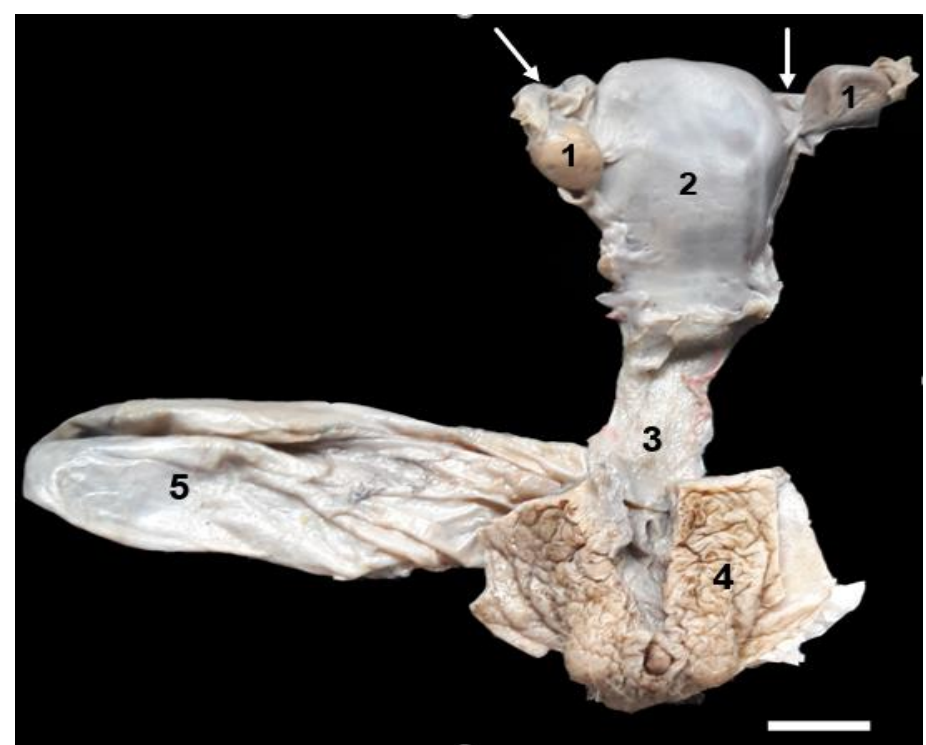

Figura 1. Imagem fotográfica da vista ventral dos órgãos reprodutores femininos de A. belzebul: 1. ovários; 2. útero; 3. vagina; 4. vulva; 5. vesícula urinária. Tubas uterinas (setas). Barra $1 \mathrm{~cm}$

Tabela 1. Dados métricos (cm) dos ovários direito e esquerdo de A. belzebul

\begin{tabular}{ccccccc}
\hline & \multicolumn{2}{c}{ Ovário esquerdo } & \multicolumn{3}{c}{ Ovário direito } \\
\cline { 2 - 7 } Animal & Comprimento & Largura & Altura & Comprimento & Largura & Altura \\
\hline 01 & 1,3 & 0,9 & 1,0 & 1,0 & 0,8 & 1,0 \\
02 & 0,9 & 0,6 & 0,6 & 0,7 & 0,5 & 0,6 \\
03 & 1,4 & 1,1 & 1,0 & 1,2 & 1,0 & 0,9 \\
04 & 0,7 & 0,6 & 0,6 & 0,6 & 0,5 & 0,5 \\
05 & 1,0 & 0,6 & 0,7 & 0,9 & 0,6 & 0,6 \\
06 & 1,2 & 1,0 & 0,9 & 1,1 & 0,8 & 0,9 \\
Média & 1,08 & 0,80 & 0,80 & 0,91 & 0,70 & 0,75 \\
DP & 0,26 & 0,22 & 0,18 & 0,23 & 0,20 & 0,20 \\
\hline
\end{tabular}

*DP = Desvio-padrão.

Fonte: Elaborada pelos próprios autores.

Após as mensurações, observou-se discreta variação de dimensão entre os ovários quando comparados entre si, sendo os ovários esquerdos maiores em $100 \%$ dos espécimes estudados, o que corrobora os achados na espécie Saimiri sciureus (Branco et al., 2010), Saguinus midas (Monteiro et al., 2012) e Sapajus libidinosus (Lima et al., 2014). Em estudos realizados com Saguinus midas, os ovários maiores são mais ativos do que os outros e, portanto, com muitos folículos em crescimento (Monteiro et al., 2012). 
Em relação à morfologia dos ovários, observaram-se diferenças anatômicas entre espécies de primatas. Em A. belzebul, verificouse que os ovários apresentam formato ovoide, dados que corroboram os encontrados em fêmeas de Cacajao calvus ucayalii (Mayor et al., 2013). Entretanto, esses dados divergem dos estudos realizados para espécies do mesmo gênero de Alouatta spp. Em Alouatta guariba clamitans (Veras et al., 2007), Alouatta caraya (Claver et al., 1984), os ovários apresentam-se morfologicamente em formato alongado e elipsoide, assim como encontrado nas espécies de primatas Saimiri sciureus (Branco et al., 2010) e Saguinus midas (Monteiro et al., 2012). Os ovários de Sapajus libidinosus são pareados, compactos, têm uma superfície lisa e forma comparável aos ovários de primatas humanos do gênero feminino, que são fusiformes durante a infância e, na fase adulta, assumem uma forma de amêndoa, medindo aproximadamente $3 \mathrm{~cm}$ de comprimento, $1,5 \mathrm{~cm}$ de largura e $1 \mathrm{~cm}$ de espessura, sendo encontrados na parede dorsal do abdome, próximos às extremidades dos cornos uterinos (Lima et al., 2014; Moore, 2014). A forma fusiforme dos ovários também é encontrada em três espécies do gênero Leontopithecus (Pissinatti et al., 2008).

$\mathrm{Na}$ anatomia microscópica do ovário de Alouatta belzebul, observou-se a presença de duas regiões, sendo: córtex e medula. A Fig. 2A mostra a região de epitélio germinativo ovariano com apenas uma única camada de células cúbicas que está aderida sobre uma camada de tecido conjuntivo denso, denominada túnica albugínea, e uma pequena parte do córtex ovariano contendo folículos em diferentes estágios de desenvolvimento. Na Fig. 2B, evidenciou-se, na área cortical ovariana, um folículo maduro (folículo de Graff) que ocupa quase todo o centro esquerdo da imagem. Esse folículo é grande e se caracteriza pelo aparecimento de um espaço intercelular denominado de antro e está preenchido por líquido folicular.Verificou-se que a grande cavidade central do folículo graafiano é circundada por diversas camadas de células granulosas. O líquido eosinofilico presente no interior da cavidade é remanescente do líquido folicular. O cúmulo oóforo projeta-se para a cavidade folicular e contém o oócito maduro não evidenciado no corte histológico.
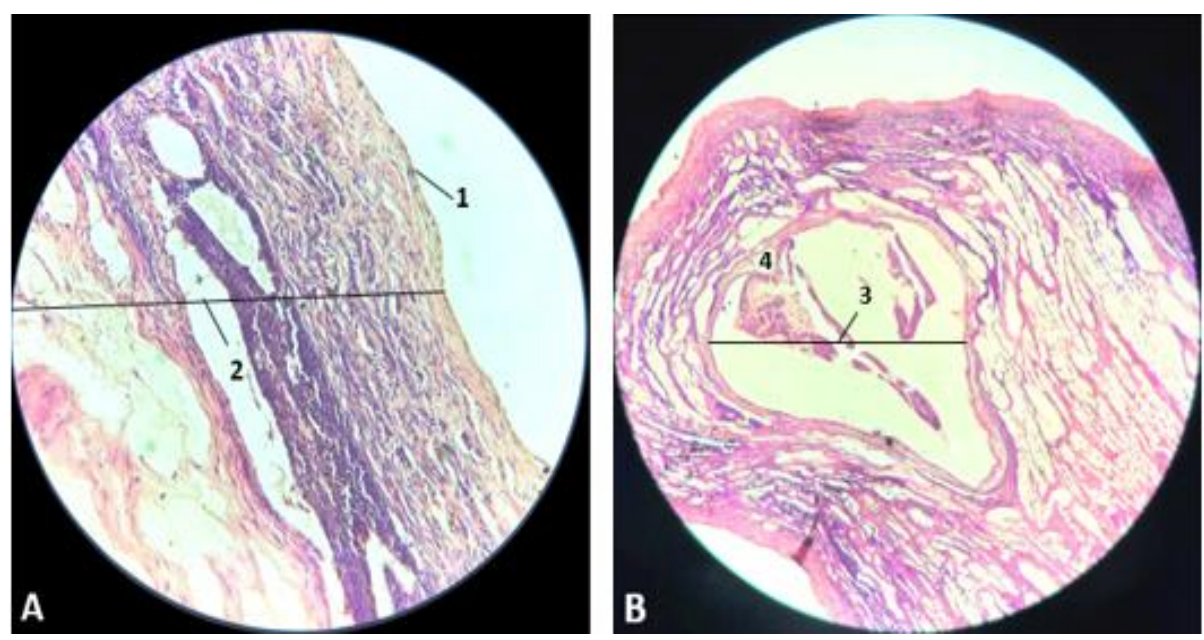

Figura 2. Análise histológica do ovário de A. belzebul. A. Fotomicrografia do ovário com aumento de 100x evidenciando apenas o epitélio germinativo ovariano (1) e parte do córtex ovariano contendo folículos em diferentes estágios de desenvolvimento (2). B. Fotomicrografia do ovário com aumento de 40x evidenciando área cortical ovariana contendo um folículo maduro (folículo graafiano) (3) e cúmulo oóforo (4).

Em Sapajus libidinosus, os folículos encontrados no córtex podem ser classificados em: primordial, primário e secundário. O primordial apresenta-se com uma camada achatada de células da granulosa ao redor do oócito; o primário é formado por uma camada de células da granulosa cuboidal; e o secundário apresenta duas ou mais camadas de células cuboidais ao redor do oócito (Lima et al., 2014). 
Em S. midas, após análise histológica, contatouse que a superfície dos ovários é coberta por tecido epitelial que apresentou variação entre o tipo cilíndrico simples a escamoso. Inferiormente a esse epitélio, observou-se uma camada de tecido conjuntivo denso, a túnica albugínea. Duas partes distintas foram identificadas no ovário: a medula e o córtex. A medula do ovário consiste em tecido conectivo solto, rico em vasos sanguíneos, com células hilares (intersticiais). O córtex ovariano apresentou-se rico em folículos ovarianos, corpo lúteo e células intersticiais (Monteiro et al., 2012). Em S. sciureus, o estudo histológico do ovário da região medular em fêmeas adultas apresentou-se de aparência parenquimatosa devido à presença de dois tipos de tecido: tecido intersticial glandular e um tecido de aparência luteinizada (Lopes et al., 2016).
Em posição lateral aos ovários, observam-se, de forma geral, em primatas, as tubas uterinas, que seguem trajetos simétricos para direita e esquerda da cavidade pélvica em direção ao útero. Em $A$. belzebul, as tubas uterinas são formadas por tubos musculares finos e curvilíneos, o que dificulta a identificação das partes descritas como infundíbulo, ampola e istmo, bem como fímbrias ovarianas. Esses achados corroboram estudos de Veras et al. (2007), em A. guariba clamitans, e de Monteiro et al. (2012), em S.midas, os quais também não evidenciaram essas partes, no entanto tais autores classificaram as fimbrias como longas, margeando o infundíbulo. A mensuração das tubas uterinas em A. belzebul mostrou comprimento médio de $2,13 \mathrm{~cm}$ para a esquerda e $2,11 \mathrm{~cm}$ para a tuba direita, conforme a Tab. 2 .

Tabela 2. Dados métricos $(\mathrm{cm})$ das tubas uterinas direita e esquerda de A.belzebul

\begin{tabular}{lcc}
\multicolumn{2}{c}{ Tuba esquerda } & Tuba direita \\
\hline Animal & Comprimento & Comprimento \\
01 & 2,4 & 2,5 \\
02 & 2,0 & 2,1 \\
03 & 2,6 & 2,3 \\
04 & 1,8 & 1,6 \\
05 & 1,9 & 2,0 \\
06 & 2,1 & 2,2 \\
Média & 2,13 & 2,11 \\
DP & 0,30 & 0,30 \\
\hline
\end{tabular}

*DP $=$ Desvio-padrão.

Fonte: Elaborada pelos próprios autores.

Em S. libidinosus, as tubas uterinas foram descritas como enroladas em espécimes adultos e retas em espécimes jovens. As mensurações também revelaram que as tubas uterinas dos espécimes jovens eram maiores do que nos espécimes adultos, com tamanho, em média, de $2,16 \mathrm{~cm}$ para as tubas uterinas direitas de fêmeas adultas, enquanto as fêmeas jovens tinham um comprimento médio de $3,10 \mathrm{~cm}$. As tubas uterinas esquerdas de fêmeas, adultas e jovens, apresentaram um comprimento médio de 2,26 e $3,85 \mathrm{~cm}$, respectivamente (Lima et al., 2014). Em fêmeas adultas da espécie $S$. midas, observou-se que as tubas uterinas eram maiores, com média de $3,5 \mathrm{~cm}$ para a tuba direita e de $3,6 \mathrm{~cm}$ para a esquerda (Monteiro et al., 2012). Nos gêneros Callithrix, Saimiri e Lagothrix, as tubas apresentaram-se retilíneas, sem distinção macroscópica de suas regiões, diferindo do grupo dos Platyrrhini e outros calitricídeos, como os
Leontopithecus, que apresentam tubas uterinas convolutas (Pissinatti et al., 2008).

$\mathrm{Na}$ anatomia microscópica de A. belzebul, evidenciou-se, nas tubas uterinas, uma camada mucosa, uma camada muscular e uma camada serosa, conforme representado na Fig. 3. Na camada mucosa, observaram-se extensas e numerosas dobras longitudinais que se projetavam no lúmen. Essa camada foi coberta por um epitélio colunar simples, sendo encontradas fibras colágenas abaixo do epitélio das dobras. Na camada muscular, foi possível observar duas camadas de músculo liso, sendo uma circular interna e outra longitudinal externa e também uma vasta vascularização. $\mathrm{Na}$ camada serosa, observaram-se células mesoteliais achatadas, um folheto visceral de peritônio com vasos sanguineos de grande calibre. 

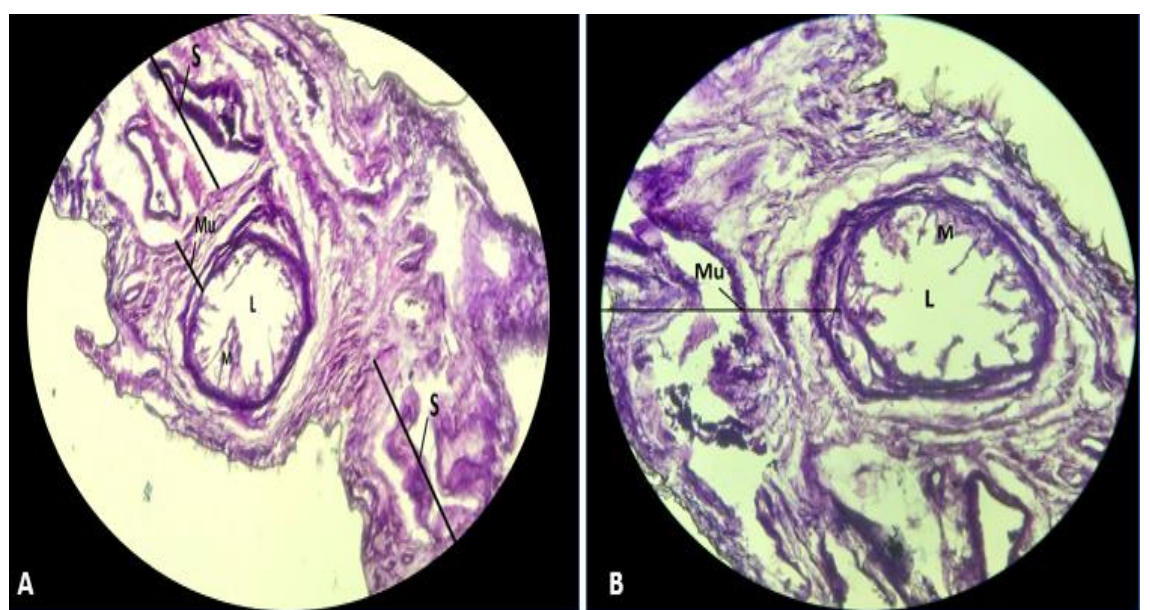

Figura 3. Análise histológica da tuba uterina do A. belzebul. A. Fotomicrografia da tuba uterina com aumento de 40x evidenciando as seguintes estruturas: lúmen (L); camada mucosa (M); camada muscular $(\mathrm{Mu})$ e a camada serosa $(\mathrm{S})$. B. Fotomicrografia da tuba uterina com aumento de 100x evidenciando somente o lúmen (L); a camada mucosa $(\mathrm{M})$; e a camada muscular $(\mathrm{Mu})$.

Em S. midas, a camada mucosa da tuba uterina é formada por epitélio colunar simples. Essa camada muscular apresenta-se muito fina, com fibras musculares lisas organizadas em grupos e separadas por abundante tecido conectivo solto. Já a camada serosa apresenta-se altamente vascularizada (Monteiro et al., 2012). Em A. belzebul, o útero é classificado como simples, semelhante à maioria dos primatas, com exceção dos prossímios, que, na maior parte do grupo, possuem dois cornos uterinos curtos.

Os primatas da subordem Anthropoidea (Catarrhini e Platyrrhini) apresentam um corpo uterino simples, com cornos uterinos ausentes, o qual é denominado de útero simples, com exceção de alguns saguis (Callithrix sp.) em que se nota uma sutil separação da extremidade cranial por um chanfro mediano. Essas características são importantes para a relação com a reprodução de primatas. Os primatas que possuem o útero bicorno têm probabilidade de gerar um número maior de filhotes por parto, enquanto aqueles que possuem o útero do tipo simples têm normalmente apenas um filhote por parto (Souza et al., 2016).

O útero de A. belzebul apresenta formato de pera invertida, de característica simples, com fundo globoso e um cérvix que se projeta na região caudal do canal vaginal. Topograficamente o ceco é bem desenvolvido em $A$. belzebul e forma um grande saco de fundo cego que funciona como uma câmara de fermentação na espécie, por conta da sua alimentação folívora, dessa forma o útero tem sua posição ligeiramente deslocada para o lado esquerdo. As medidas do comprimento do útero entre os animais estudados variaram de 3,1 a $4,8 \mathrm{~cm}$, conforme a Tab. 3 .

Tabela 3. Dados métricos (cm) do útero de $A$. belzebul

\begin{tabular}{cc} 
& Útero \\
\hline Animal & Comprimento \\
01 & 4,8 \\
02 & 4,4 \\
03 & 3,7 \\
04 & 3,1 \\
05 & 3,4 \\
06 & 3,6 \\
Média & 3,83 \\
DP & 0,64 \\
\hline
\end{tabular}

*DP = Desvio-padrão.

Fonte: Elaborada pelos próprios autores.

O tamanho do útero também apresenta ampla variabilidade nas diferentes espécies. Em $A$. belzebul, mensuraram-se $3,83 \mathrm{~cm}$ de média entre o fundo até o cérvix. Semelhante a esses achados, há os resultados encontrados na Macaca mulatta, na qual o comprimento total do útero nas fêmeas nulíparas teve média de $4,12 \mathrm{~cm}$, nas primíparas de $4,32 \mathrm{~cm}$ e nas pluríparas de $5,14 \mathrm{~cm}$ (Souza et al., 2016). Em contrapartida, na espécie S.midas, o tamanho do útero é proporcionalmente pequeno, medindo aproximadamente $1,4 \mathrm{~cm}$. (Monteiro et al., 2012). 
Essa variação pode ser grande mesmo em espécies do mesmo gênero, como é o caso do gênero Saimiri. Na espécie $S$. Sciureus, a distância do fundo até o óstio externo varia de 1,5 a $2,8 \mathrm{~cm}$, contudo, em $S$. Collinsi, essa mesma distância chega à média de $6,3 \mathrm{~cm}$ (Lopes et al., 2016). Essa diferença de tamanhos do útero em espécies do mesmo gênero ocorre também entre A. guariba clamitans, que apresenta comprimento médio do útero de 2,8cm (Veras et al., 2007), sendo mais semelhante ao útero de uma espécie de outro gênero, o $S$. Libidinosus, que apresenta $2,36 \mathrm{~cm}$ de média (Lima et al., 2014), do que de A. belzebul, que faz parte do mesmo gênero.

No útero de A. belzebul, após análise histológica, evidenciaram-se as suas três camadas. Na Fig. 4, observa-se o endométrio e parte do miométrio. $\mathrm{O}$ endométrio (membrana mucosa uterina) é revestido por epitélio colunar cilíndrico simples e por algumas glândulas tubulares. O miométrio apresentou-se como sendo a camada mais espessa do útero de A. belzebul, organizada em feixes de fibras musculares lisas com colágeno intercalado e orientação aleatória, com uma alta vascularização. O padrão aleatório de células musculares lisas do miométrio está associado ao papel fundamental das contrações desses músculos durante o período em que a fêmea está prenha e no parto, sendo regulado por fatores hormonais e moleculares (Lopes et al., 2016). O perimétrio é a camada mais externa das três camadas, constituído por uma fina camada de tecido conjuntivo que se encontra aderida ao miométrio.

Em A. belzebul, identificou-se a vagina como um canal longo, achatado dorsoventralmente, com comunicação caudal com o vestíbulo da vagina e com correlação cranial com o cérvix; no canal vaginal, não foram observadas as pregas da mucosa vaginal. Esses dados corroboram a maioria dos primatas do Novo Mundo. Entretanto, em S.libidinosus, as pregas longitudinais foram visualizadas na mucosa do canal vaginal (Lima et al., 2014). Em A. guariba clamitans (Veras et al., 2007) e em S. sciureus (Branco et al., 2010), a dissecação da vagina revelou uma mucosa com pregas longitudinais em toda a sua extensão, porém, nos Leontopithecus (Pissinatti et al., 2008), a mucosa vaginal apresenta um pregueamento disposto transversalmente por toda sua extensão.

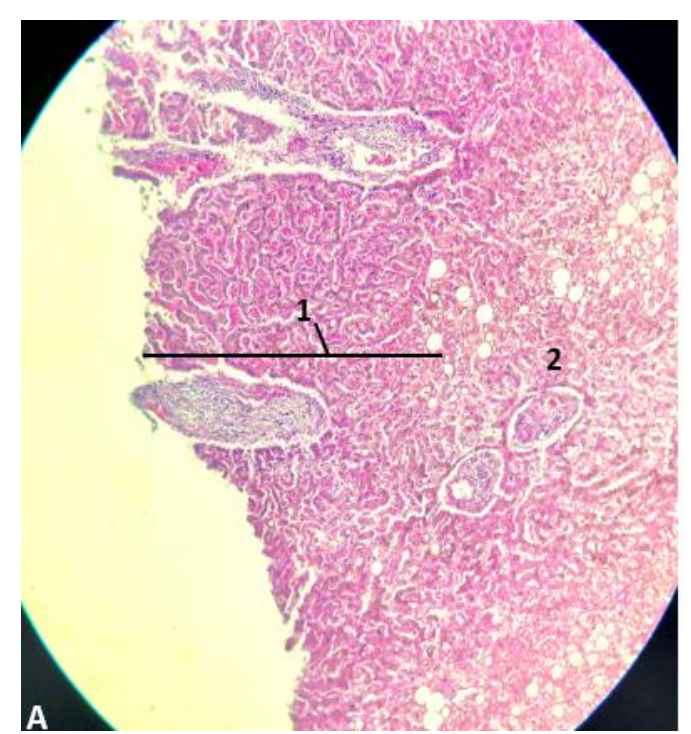

Figura 4. Análise histológica do útero de $A$. belzebul. Fotomicrografia do útero com aumento de $100 x$ evidenciando a camada endometrial (1) e parte da camada miometrial (2).

Em A. belzebul, mensurou-se o a vagina a partir do vestíbulo até a união com o útero, uma média de $3,8 \mathrm{~cm}$ de comprimento, dados que se aproximam ao encontrado em $A$. guariba clamitans (Veras et al., 2007), que apresentou $3,35 \mathrm{~cm}$ de comprimento. Contudo, em $S$. midas (Monteiro et al., 2012), a vagina apresentou um comprimento médio de $2,6 \mathrm{~cm}$ e, em $S$. libidinosus (Lima et al., 2014), a vagina apresentou $2,13 \mathrm{~cm}$ de comprimento, o que difere do encontrado em S. sciureus (Branco et al., 2010), de 1,3cm. Em humanos do gênero feminino, a vagina apresenta de 7 a $9 \mathrm{~cm}$ de comprimento, com espessura média de 3mm (Dangelo; Fattini, 2011).

$\mathrm{Na}$ anatomia microscópica da vagina de A.belzebul (Fig. 5A), observou-se que a camada muscular da parede vaginal apresenta músculo liso disposto em duas camadas mal definidas, e, além da vagina, verifica-se a uretra e seu lúmen. A mucosa vaginal é composta por epitélio estratificado pavimentoso não queratinizado atrófico e por tecido conjuntivo denso não modelado (Fig. 5B e 5C). 


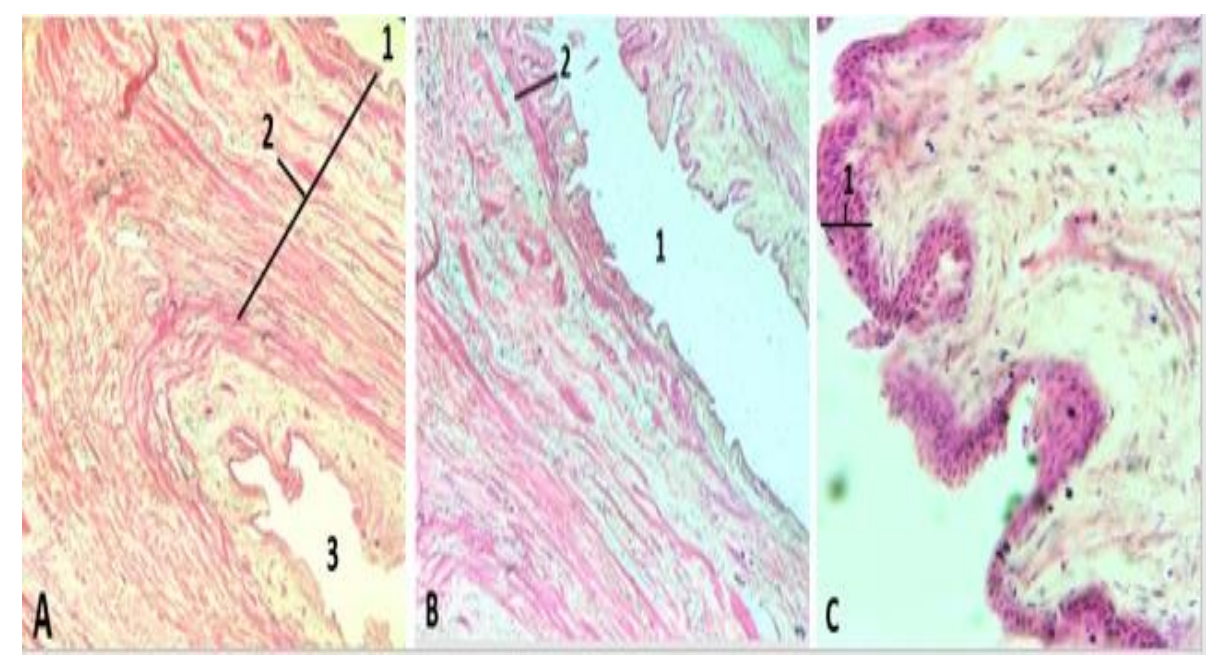

Figura 5. Análise histológica da vagina de A. belzebul. A. Fotomicrografia da vagina com aumento de 40x evidenciando o lúmen vaginal (1), a camada muscular da parede vaginal (2) e o lúmen da uretra (3). B. Fotomicrografia da vagina com aumento de 100x evidenciando o lúmen vaginal (1) e a mucosa vaginal (2). C. Fotomicrografia da vagina com aumento de 400x evidenciando a mucosa vaginal (1).

Durante a análise histológica da vagina de $S$. Libidinosus (Lima et al., 2014), o epitélio não pôde ser classificado devido à autólise. O epitélio vaginal da fêmea de primatas não humanos, como A. guariba clamitans e A. caraya, foi classificado como escamoso estratificado queratinizado, sem pregas longitudinais (Veras et al., 2007). O vestíbulo é como um canal muscular longo e interno que comunica a vagina com a vulva. No entanto, alguns autores que descreveram o aparelho genital feminino de primatas consideraram esse segmento do trato genital como uma porção da vagina (Monteiro et al., 2012; Lima et al., 2014).

As configurações da terminação da vagina variam muito entre os Platyrrhini. $\mathrm{Na}$ maioria dos primatas, o óstio uretral apresenta-se situado em uma papila distinta no vestíbulo vaginal, no entanto a uretra feminina do A.belzebul tem origem no trígono vesical e desemboca no vestíbulo da vagina, criando uma papila bilobada, conforme o que pode ser evidenciado na seta abaixo (Fig. 6).
Esses resultados corroboram os encontrados em fêmeas de A. guariba clamitans em estudos de Veras et al. (2007), que identificaram o óstio externo da uretra com abertura na parede ventral da vagina em uma papila distinta, proeminente e bilobada, no entanto essa abertura ocorre na região medial da vagina (acima do vestíbulo), diferentemente do que foi encontrado em $A$. belzebul, na qual a abertura está localizada na região vestibular da vagina. Em S. libidinosus (Lima et al., 2016), o sistema reprodutor feminino apresentou uma uretra bastante curta, com origem no trígono vesical, que também desembocava no vestíbulo da vagina, todavia, por meio do óstio uretral externo, já em Leontopithecus (Pissinatti et al., 2008), a uretra inicia-se no terço final da vagina, como um tubo muscular percorrendo a parede ventral, abrindo-se como um óstio no lúmen vaginal.

Em A. belzebul, evidenciou-se a vulva caracterizada por um epitélio altamente pregueado, rugoso, com poucos e espassados pelos. A vulva se encontra disposta ventralmente ao clitóris, que é bem desenvolvido (hipertrófico) (Fig. 7), corroborando os dados encontrados em $S$. libidinosus (Lima et al., 2014). 


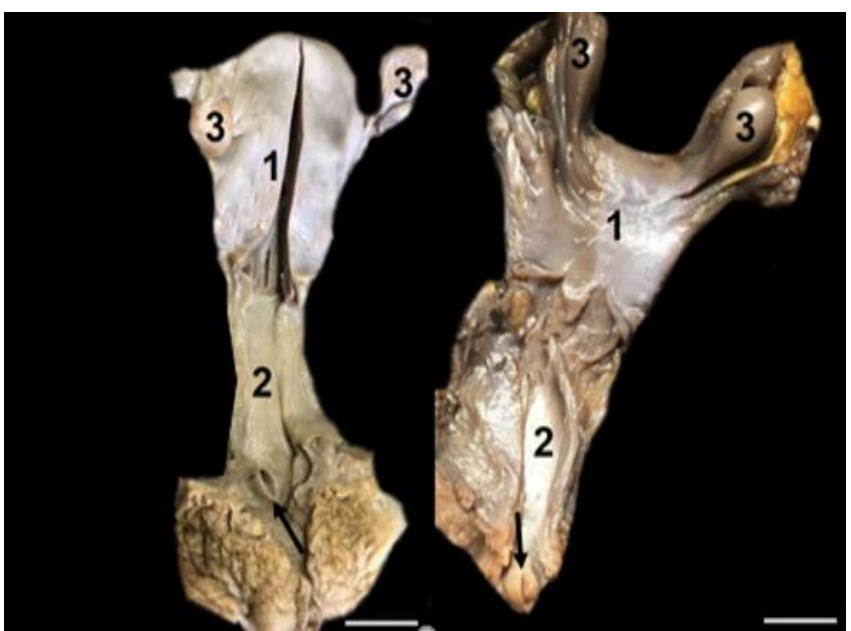

Figura 6. Imagem fotográfica da vista ventral dos órgãos reprodutores femininos de A. belzebul evidenciando: 1: útero; 2: vagina; 3: ovários; papila uretral bilobada (seta). Barra: $1 \mathrm{~cm}$.

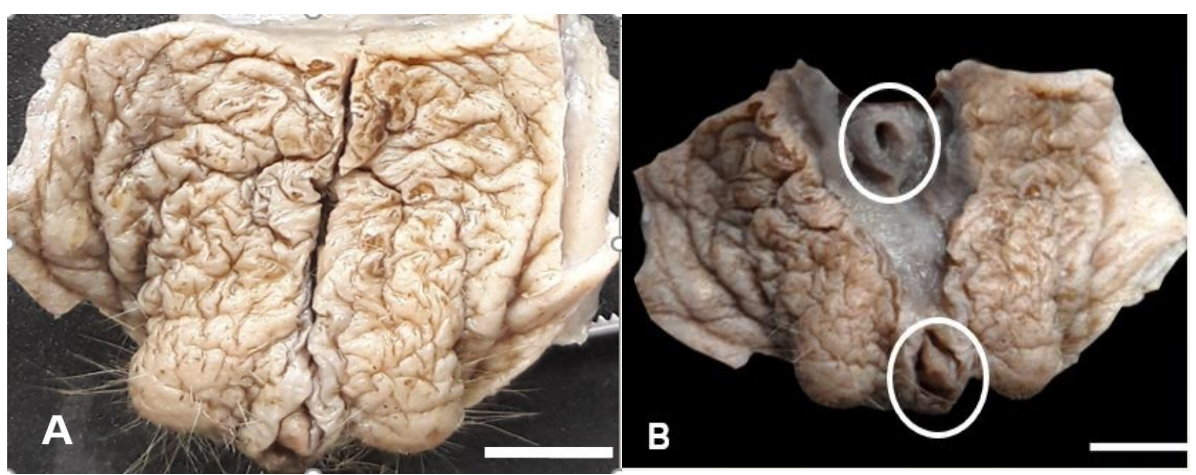

Figura 7. A. Imagem fotográfica da vista ventral da genitália externa de A. belzebul. B. Abertura da região vulvar para visualização dos óstios externo da uretra e vaginal (círculo branco superior) e o grande clitóris (círculo branco inferior). Barra $1 \mathrm{~cm}$.

Em $S$. midas, essa característica é diferente, pois o clitóris praticamente não é evidenciado, estando totalmente coberto pelos lábios menores (Monteiro et al., 2012). O clitóris de Ateles é descrito como particularmente longo, dos Callitrichidae como discretos, o de Sapajus e o de Lagothrix são descritos como desenvolvidos e pendentes (Porto et al., 2010). O clitóris de algumas espécies de primatas das famílias Cebidae e Atelidae pode, em alguns casos, ser maior que o pênis dos machos da mesma espécie (Souza et al., 2016). Na grande maioria dos primatas, os grandes lábios estão geralmente ausentes, sendo encontrados somente na infraordem Prosimii e na família Cebidae (Monteiro et al., 2012; Lima et al., 2014).

Os órgãos genitais externos de primatas são usualmente utilizados para cópula, excreção, reconhecimento, estímulos sexuais e marcação territorial, ou seja, é muito importante para a comunicação visual em relação ao comportamento sexual em primatas não humanos. Periodicamente, a genitália externa, sobretudo das fêmeas de várias espécies, desenvolve-se de forma excepcional, tornandose proeminente e atraente aos cinco sentidos, incluindo o paladar, em alguns casos, para o sexo oposto (Andrade et al., 2010).

\section{CONCLUSÃO}

Os achados morfológicos descritos neste trabalho apresentam, pela primeira vez, a descrição da anatomia do sistema reprodutor feminino de $A$. belzebul. Diante dos objetivos delineados e dos resultados alcançados, é possível concluir que a espécie A. belzebul apresenta órgãos genitais femininos similares aos das outras espécies de primatas não humanos, mostrando particularidades morfológicas próprias da espécie. 
As comparações neste estudo também mostraram que, mesmo em indivíduos pertencentes à mesma família e ao mesmo gênero, há diferenças no sistema reprodutor feminino. Dessa forma, a presente pesquisa fundamenta-se na necessidade de se obterem informações que possam subsidiar novos estudos, principalmente no que concerne à anatomia do sistema reprodutor feminino de primatas não humanos.

\section{REFERÊNCIAS}

ANDRADE, A.; ANDRADE, M.C.R.; MARINHO, A.M. et al. Biologia, manejo e medicina de primatas não humanos na pesquisa biomédica. Rio de Janeiro: Fiocruz, 2010. 471p.

BENNETT, B.T.; ABEE, C.R.; HENRICKSON, R. Nonhuman primates in biomedical research: biology and management. San Diego: Academic Press, 1995. v.1, 512p.

BRANCO, E.; LACRETA JUNIOR, A.C.C.; ISHIZAKI, M.N. et al. Morfologia macroscópica e morfometria do aparelho urogenital do macaco de cheiro (Saimiri sciureus Linnaeus, 1758). Biotemas, v.23, p.197-202, 2010.

CLAVER, J.A.; LAWZEWITSC, I.; COLILLAS, O.J. Microscopic anatomy of the ovary of Alouatta caraya. Primates, v.3, p.362-371,1984.

DANGELO, J.G.; FATTINI, C.C. Anatomia sistêmica e segmentar. 3.ed. São Paulo: Atheneu, 2011. 780p.

DIXON A.F. Callitrichid mating systems: laboratory and field approaches to studies of monogamy and polyandry. In: RYLANDS, A.B. (Ed.). Marmosets and tamarins: systematics, behaviour and ecology. Oxford: Oxford University Press, 1994. p.164-175.

DIXON A.F. The ovarian cycle and sexual behaviour. In: ___ (Ed.). Primate sexuality: comparative studies of the prosimians, monkeys, apes, and human beings. Oxford: Oxford University Press, 1998. p.315-355

GUIMARÃES, M.A.B.V. Reprodução de primatas não-humanos. Rev. Bras. Reprod. Anim., v.31, p.339-343. 2007.

LIMA, A.R.; GUIMARÃES, S.B.; BRANCO, É. et al. Morphological and morphometric description of female reproductive tract of Sapajus apella (Capuchin monkey). Anat. Histol. Embryol., v.44, p.262-268, 2014.
LIMA, A.R.; GUIMARÃES, S.B.; BRANCO, É. et al. Anatomy and histology of the urinary tract in the capuchin monkey (Sapajus apela). Pesqui. Vet. Bras., v.36, p.221-226, 2016.

LOPES G.P.; BRITO, A.B.; PAIM, F.P. et al. Comparative characterization of the external genitalia and reproductive tubular organs of three species of the genus Saimiri Voigt, 1831 (Primates: Cebidae). Anat. Histol. Embryol., v.46, p.143-161, 2016.

MAYOR, P.; BOWLER, M.; LOPEZ, A.P.C. Functional morphology of the female genital organs in the peruvian red uakari monkey (Cacajao calvus ucayalii). Am. J. Primatol., v.75, p.545-554, 2013.

MONTEIRO, N.C.; LIMA, A.R.; CARVALHO, A.F. et al. Morphology and morphometry of the reproductive system of female Saguinus midas (Linnaeus, 1758). Microsc. Res. Tech., v.75, p.720-726, 2012.

MOORE, K.L. Anatomia orientada para a clínica. 7.ed. Rio de Janeiro: Guanabara Koogan, 2014. 1128p.

PISSINATTI, L.; TORTELLY, R.; PORTO, M. et al. Morfologia macroscópica do aparelho reprodutor feminino de Leontophitecus cativos (Lesson, 1840) primates callitrichidae. Arq. Bras. Med. Vet. Zoote., v.60, p.1472-1475, 2008.

PORTO, M.; PISSINATTI, A.; BURITY, C.H.F. et al.Morphological description of the clitoris from the Leontopithecus rosalia (Linnaeus, 1766), leontopithecus chrysomelas (Kuhl, 1820) and Leontopithecus chrysopygus (Mikan, 1823) (primates, platyrrhini, callitrichidae) Ann. Nat. Med., v.180, p.1-9, 2010.

SOUZA. I.V.; KUGELMEIER, T.; ANDRADE, M. et al. Aspectos morfológicos do útero de macaco rhesus (Macaca mulatta - Zimmermann, 1780) em fêmeas nulíparas, primíparas e plurípara. Rev. Soc. Bras. Ciênc. Anim. Lab., v.4, p.15-21, 2016.

VERAS, M.M.; MIGLINO, M.A.; SILVA, Z. Morfologia do aparelho reprodutor em fêmeas de bugio marrom (Alouatta guariba clamitans). Braz. J. Vet. Res. Anim. Sci., v.44, p.12-17, 2007. 